\title{
MALFORMACIONES CONGÉNITAS DEL OÍDO EXTERNO Y MEDIO EN PACIENTES DE LA CÁTEDRA DE OTORRINOLARINGOLOGÍA, FACULTAD DE CIENCIAS MÉDICAS, UNIVERSIDAD NACIONAL DE ASUNCIÓN, PARAGUAY
}

\author{
CONGENITAL MALFORMATIONS OF THE EXTERNAL AND MEDIUM EAR IN PATIENTS \\ OF THE OTORHINOLARYNGOLOGY DEPARTMENT, SCHOOL OF MEDICAL SCIENCES, \\ NATIONAL UNIVERSITY OF ASUNCIÓN, PARAGUAY
}

Wilma VILLAMAYOR ${ }^{1}$, Oscar RUIZ-DÍAZ ${ }^{1}$, Karen ZÁRATE ${ }^{1}$, Mario SAUCEDO ${ }^{1}$, Laura TANDI ${ }^{1}$, Pedro SÁNCHEZ ${ }^{1}$, Jorge ROIG ${ }^{2}$.

${ }^{1}$ Estudiantes de Medicina y Cirugía, Facultad de Ciencias Médicas, Universidad Nacional de Asunción, San Lorenzo - Paraguay

${ }^{2}$ Profesor Adjunto de Otorrinolaringología, Facultad de Ciencias Médicas, Universidad Nacional de Asunción, San Lorenzo - Paraguay.

Cómo citar este artículo: Villamayor W, Ruiz-Díaz O, Zárate K, Saucedo M, Tandi L, Sánchez P, et al. Malformaciones congénitas del oído externo y medio en pacientes de la Cátedra de Otorrinolaringología, Facultad de Ciencias Médicas, Universidad Nacional de Asunción, Paraguay. Medicina Clínica y Social. 2017;1(2):105-113.

\section{RESUMEN}

Introducción: Los recién nacidos tienen una alta incidencia de deformidades del oído, causadas por las interacciones de una serie de factores. La ubicación, los síntomas clínicos, los hallazgos de imágenes y un alto índice de sospecha ayudan al diagnóstico de las malformaciones del oído. Las estructuras del oído medio y del externo proceden del primer y del segundo arcos branquiales. Como las mismas se encuentran relacionadas ya desde su origen, así también lo pueden estar sus alteraciones. Objetivo: El presente trabajo se realizó para conocer la frecuencia de las malformaciones congénitas del oído en nuestro medio y, además, con el objetivo de que sea una base para futuras investigaciones sobre el tema. Metodología: Estudio observacional descriptivo transverso, con muestreo no probabilístico de casos consecutivos, llevado a cabo en la Cátedra y Servicio de Otorrinolaringología de la Facultad de Ciencias Médicas, Universidad Nacional de Asunción, en junio de 2017. Se analizaron 5781 historias clínicas de pacientes que consultaron a la Unidad de Otología, entre enero 2006 a mayo 2017. Las variables exploradas fueron: edad (en años cumplidos), sexo (masculino, femenino), motivo de consulta y diagnóstico. Los datos fueron analizados utilizando estadística descriptiva. Resultados: Se encontró una frecuencia de 1,07\% de malformaciones congénitas del oído externo y medio, lo que representa un total de 63 pacientes. De los 63 pacientes con malformaciones congénitas, el 52\% era del sexo femenino (33 pacientes), con edades comprendidas entre 1 y 70 años (media: 26,42). El diagnóstico más prevalente fue el de fistula auris (33\%). Los motivos de consultas más frecuentes fueron la tumoración cervical (32\%) y la hipoacusia (25\%). Discusión: Resulta importante tanto para el pediatra como para el otorrinolaringólogo lograr identificar precozmente las malformaciones congénitas del oído, para así poder instaurar terapéuticas oportunas y acertadas, que mejoren ampliamente la calidad de vida de los pacientes.

Palabras clave: Malformaciones congénitas; Oído externo; Oído medio. 


\section{ABSTRACT}

Introduction: Newborns have a high incidence of ear deformities, caused by the interactions of a number of factors. Location, clinical symptoms, imaging findings, and a high index of suspicion help diagnose ear malformations. The structures of the middle and external ear come from the first and second gill arches. As they are related already since its origin, so can their alterations. Objective: The present work was carried out to know the frequency of congenital malformations of the ear in our environment and, in addition, with the objective of being a basis for future research on the subject. Methodology: It was a transversal, descriptive, observational study, with non-probabilistic sampling of consecutive cases, carried out at the Department of Otorhinolaryngology, School of Medical Sciences, National University of Asunción, in June 2017. We analyzed 5781 medical records of patients (January 2006 to May 2017). The variables studied were: age (in years completed), sex (male, female), reason for consultation and diagnosis. Data were analyzed using descriptive statistics. Results: A frequency of $1.07 \%$ of congenital malformations of the external and middle ear was found, representing a total of 63 patients. Of the 63 patients with congenital malformations, 52\% were female (33 patients), aged between 1 and 70 years (mean: 26.42). The most prevalent diagnosis was fistula auris (33\%). The most frequent reasons for consultation were cervical tumor (32\%) and hearing loss (25\%). Discussion: It is important for both the pediatrician and the otolaryngologist to identify prematurely the congenital malformations of the ear, in order to establish timely and accurate therapies that could greatly improve the quality of life of patients.

Keywords: Congenital malformations; External ear; Middle ear.

\section{INTRODUCCIÓN}

Las malformaciones del oído, según la clasificación de Bellucci (1981), se dividen en cinco grupos: fístulas preauriculares y quistes, malformaciones del primer surco branquial: duplicación del conducto auditivo externo (tipo I: solamente ectodermo y tipo II: ectodermo y mesodermo), anomalías del primero y segundo arcos branquiales: no envolviendo el conducto auditivo externo ni membrana timpánica, deformidades del surco branquial no envolviendo los osículos: hipoplasia del hueso timpánico y falla en la reabsorción en el conducto auditivo externo, microtia, agenesia del conducto auditivo externo, y malformación de la membrana timpánica y cadena osicular, con o sin deformidad de la cara (1).

Los arcos branquiales son los precursores embriológicos de la cara, el cuello y la faringe. Las anomalías de los arcos branquiales son las segundas lesiones congénitas más frecuentes de la cabeza y el cuello en los niños, siendo las anomalías del segundo arco branquial de lejos las más comunes. Clínicamente, estas anomalías congénitas pueden presentarse como quistes, tractos sinusales, fístulas o restos cartilaginosos con hallazgos clínicos y radiológicos típicos.

La ubicación, los síntomas clínicos, los hallazgos de imágenes y un alto índice de sospecha ayudan al diagnóstico de las malformaciones del oído (2). Las estructuras del oído medio y del externo proceden del primer y del segundo arcos branquiales (3). Como las mismas se encuentran relacionadas ya desde su origen, así también lo pueden estar sus alteraciones.

Las malformaciones del oído externo y del oído medio son predominantemente unilaterales (alrededor del 70-90\%) y en su mayoría afectan al oído derecho. La incidencia de malformaciones del oído es de aproximadamente 1 en 3800 recién nacidos. Los pasos diagnósticos incluyen examen clínico, pruebas audiológicas, análisis genético y, especialmente, pruebas radiológicas. La descripción precisa de las malformaciones mediante la tomografía computada 
(TC) y la resonancia magnética (RM) es indispensable para la planificación y el éxito de los procedimientos de reconstrucción y rehabilitación del oído quirúrgico (4).

Combinaciones típicas y menos típicas de las malformaciones del oído pueden derivarse de la embriogénesis. Los signos clínicos y los hallazgos audiométricos conducen al diagnóstico en las disgenesias del oído. En las malformaciones del oído externo y del oído medio, la TC es la modalidad de imagen elegida (5).

Los recién nacidos tienen una alta incidencia de deformidades auriculares, causadas por las interacciones de una serie de factores. Por ejemplo, la incidencia de deformidad auricular neonatal en el área del delta del río de las Perlas, China, se encuentra alrededor del 57,46 por ciento. Esta alta incidencia se explica por la presencia de siete factores estadísticamente significativos: situación de parto de la madre, antecedentes de hepatitis materna, embarazo anormal, trabajo anormal, desproporción cefalopélvica, rotura prematura de membranas y peso neonatal (6).

La malformación congénita del oído externo y medio tiene una influencia absoluta sobre el estado psicológico de los pacientes, pero su efecto es sólo alrededor del $15 \%$ de los factores influyentes multitudinarios. Y los pacientes tienen, además, un alto riesgo de trastornos del comportamiento (7). La atresia del conducto auditivo externo es $70 \%$ unilateral, con afectación más frecuente en hombres y del lado derecho (8).

Atresia o estenosis congénita del conducto auditivo externo sin malformación del oído externo o microtia es poco común. Pocos informes de esta malformación se publicaron antes de los años ochenta. Una serie de 311 pacientes con atresia del conducto auditivo externo, reportada por Jafek y colaboradores, mostró que sólo 24 (alrededor del 6\%), tenían atresia sin una microtia concomitante (9). Grundfast y Camilon examinaron 10 casos de la literatura mundial, corroborando que la ausencia de una evidente malformación externa obstaculiza la detección temprana, haciendo que la edad media del diagnóstico fuera de 2,5 años, con un rango de 2 meses a 7 años (8). Por su parte, la microtia-anotia tiene una gran connotación para la vida del individuo, con problemas funcionales y psicológicos asociados, lo que obliga a tratamientos especializados y multidisciplinarios (10).

175 malformaciones y 303 deformidades del oído infantil fueron tratadas con el Sistema EarWell. Este sistema demostró ser eficaz para eliminar o reducir la necesidad de cirugía en todas las malformaciones, excepto las más graves (11).

El presente trabajo se realizó debido a la importancia de conocer la frecuencia de las malformaciones del oído en nuestro medio y, además, con el objetivo de que sea una base para futuras investigaciones sobre el tema en Paraguay.

\section{METOdOLOGÍA}

Fue un estudio observacional descriptivo transverso, con muestreo no probabilístico de casos consecutivos, Ilevado a cabo en la Cátedra y Servicio de Otorrinolaringología de la Facultad de Ciencias Médicas, Universidad Nacional de Asunción, en junio de 2017. Se analizaron 5781 historias clínicas de pacientes que consultaron a la Unidad de Otología, entre enero 2006 a mayo 2017. Se excluyeron aquellas historias clínicas incompletas. 
Las variables exploradas fueron: edad (en años cumplidos), sexo (masculino, femenino), motivo de consulta y diagnóstico. En todo momento se resguardó la identidad de los pacientes y se respetaron los principios de la ética en la investigación.

Los datos fueron recolectados previa autorización del Jefe de Cátedra y Servicio de Otorrinolaringología y se archivaron utilizando tablas de Microsoft Excel ${ }^{\circledR}$. Posteriormente, fueron analizados con el software estadístico Epilnfo versión 7.2 (CDC, Atlanta), utilizando estadística descriptiva. Se garantizó el anonimato de los datos personales de los pacientes, los cuales no fueron incluidos en los resultados de la investigación.

\section{RESULTADOS}

La muestra estuvo conformada por 5871 historias clínicas de pacientes. Se encontró una frecuencia de $1,07 \%$ de malformaciones congénitas del oído externo y medio, lo que representa un total de 63 pacientes.

De los 63 pacientes con malformaciones congénitas, el 52\% era del sexo femenino (33 pacientes), con edades comprendidas entre 1 y 70 años (media: 26,42). La distribución diagnóstica de los 63 pacientes puede verse en la figura 1.

Figura 1. Distribución diagnóstica de malformaciones congénitas del oído externo y medio ( $n=63)$.

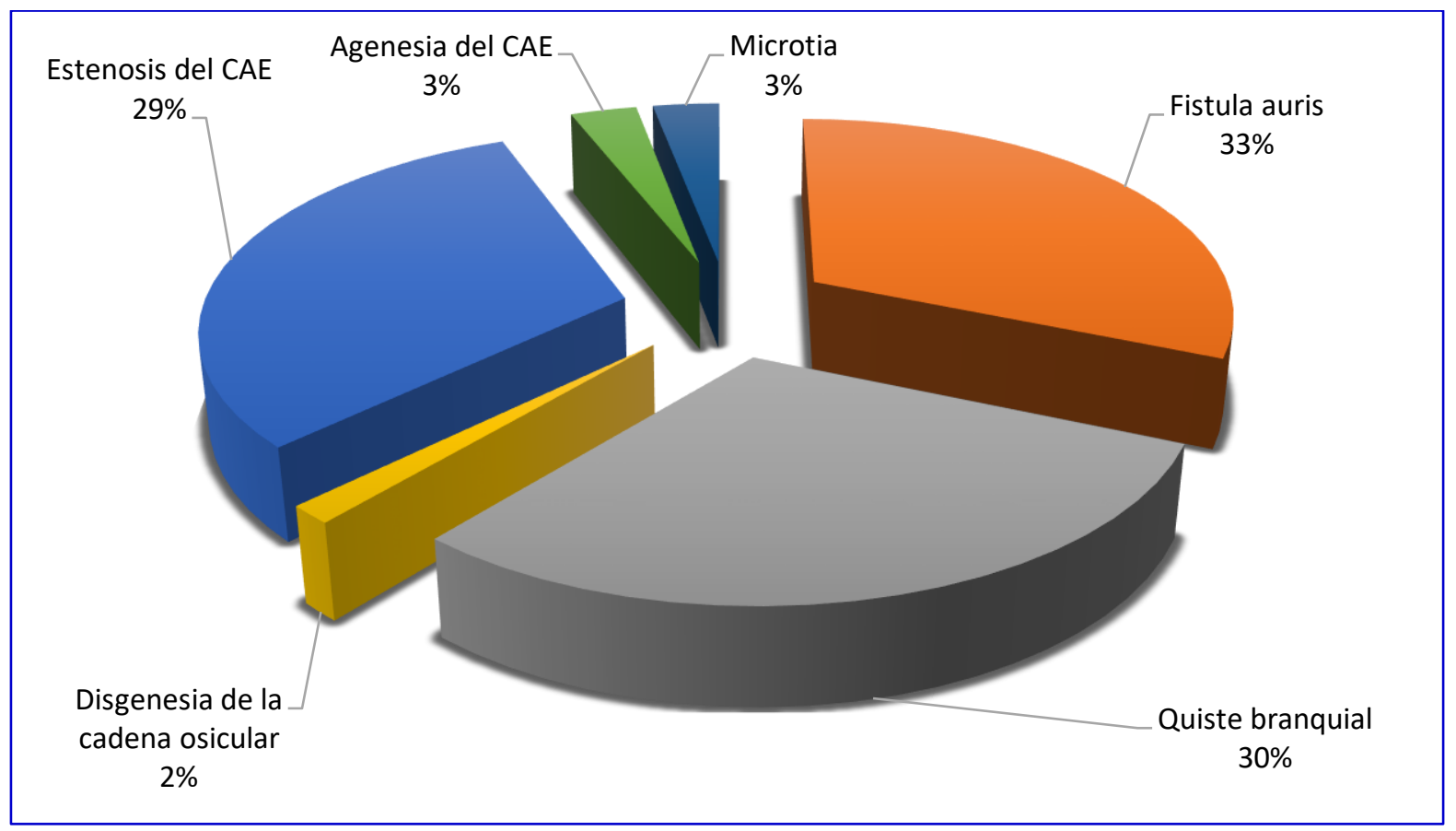

El motivo de consulta más frecuente fue la tumoración cervical (32\%), seguido en frecuencia por la hipoacusia (25\%) (Tabla 1). 


\begin{tabular}{|c|c|}
\hline \begin{tabular}{|} 
TABLA 1. MOTIVOS DE CONSULTA POR MALFORMACIONES \\
CONGÉNITAS DEL OÍDO EXTERNO Y MEDIO
\end{tabular} \\
\hline Motivo de consulta & Frecuencia (n=63) \\
\hline Tumoración cervical & $32 \%$ \\
Hipoacusia & $25 \%$ \\
Otorrea & $23 \%$ \\
Tumoración submaxilar & $10 \%$ \\
Nódulo cervical & $3 \%$ \\
Secreción preauricular & $3 \%$ \\
Tumoración preauricular & $2 \%$ \\
Tumoración subauricular & $2 \%$ \\
\hline
\end{tabular}

\section{DISCUSIÓN}

Teniendo en cuenta que la fístula auris en general es asintomática, fue un hallazgo interesante el hecho de que la misma fue la malformación congénita de oído más frecuentemente diagnosticada en Unidad de Otología de la Cátedra y Servicio de Otorrinolaringología de la Facultad de Ciencias Médicas de la Universidad Nacional de Asunción, en el periodo de tiempo comprendido entre enero de 2016 y mayo de 2017.

La frecuencia de fistula auris oscila entre $1,9 \%$ y $5 \%(12,13)$. Esto contrasta con los hallazgos de nuestra investigación, que informan de una frecuencia del 33\%. Aunque por lo general los pacientes que requerirán de cirugía por fístula auris son la excepción, en nuestro estudio se reportó que 21 casos requirieron de manejo quirúrgico. Aquí reside la importancia de conocer y saber llevar a la práctica las diferentes técnicas disponibles para tratar las complicaciones de la fístula auris, teniendo en consideración las opciones quirúrgicas disponibles y válidas (14).

Asimismo, otro punto a tener en cuenta es el hecho de que las fístulas auriculares pueden estar asociadas a otras malformaciones, como las renales. No obstante, en un estudio realizado por Melnick con 58,000 casos con defectos auriculares, se encontraron 446 pacientes con fístula preauricular y de estos, ninguno tuvo anomalías renales y sólo 15 se asociaron con algún síndrome genético (15). En esta investigación ninguno de los pacientes con fístula auris se asoció con alguna otra malformación, aunque no se descarta la posibilidad de realizar estudios complementarios que ayuden a certificar la falta de las mismas.

El quiste branquial de origen congénito de localización cervical es, con frecuencia, motivo de consulta, lo que se relaciona favorablemente con nuestro estudio al ser el motivo de consulta más frecuente en las historias clínicas analizadas con $32 \%$ del total de los motivos de consulta. Quintana y colaboradores evidenciaron el comportamiento de los quistes branquiales en el Hospital "Ciro Redondo García", de Artemisa (Cuba), en el periodo comprendido entre los años 1993 al 2009, y registraron 12 quistes branquiales, que afectaron a ambos sexos por igual, un $75 \%$ a pacientes entre 15 y 30 años y un $66,7 \%$ afectó el lado derecho del cuello (16). En 
nuestro trabajo encontramos 19 quistes branquiales, con una leve diferencia a favor del sexo femenino (60\%) y con una media de edad de 34 años.

Es importante recalcar la diferencia en la procedencia de los quistes branquiales de primer y segundo arco branquial, la cual en nuestro estudio mostró un 55\% para los de primer arco y de $45 \%$ para los del segundo. Esto discrepa con el estudio de Medina Vega y colaboradores que mostró que entre un $92 \%$ y un $90 \%$ se relacionan con el segundo arco branquial (17). Consideramos esencial seguir complementando más estos estudios, ya que en varias ocasiones se presentan dificultades al establecer un diagnóstico presuntivo certero en casos de tumores quísticos cervicales aún después de seguir toda una serie de pasos y estudios complementarios. Entrada Sarmientos (18) y Santana Garay (19) reconocen al quiste tirogloso como el quiste malformativo cervicofacial de mayor prevalencia. Cabe recordar aquí que ante una tumoración cervical se ha de establecer el diagnóstico diferencial con tres etiologías posibles: congénita, infecciosa y neoplásica (20).

Como se mencionó previamente, la atresia congénita del conducto auditivo externo sin malformación del oído externo o microtia es poco común. En el presente estudio encontramos un porcentaje de 3,2\% ( 2 casos) de atresia o agenesia congénita del conducto auditivo externo sin malformación asociada. La edad media al momento del diagnóstico en nuestros pacientes fue de 31,8 (rango de 6 a 70 años), lo que contrasta con el estudio de Grundfast y Camilon, quienes encontraron una edad media de 2,5 años (rango de 2 meses a 7 años) (8). Esta diferencia podría deberse a la consulta tardía por parte de nuestros pacientes.

La estenosis aural congénita conlleva un riesgo mucho mayor de colesteatoma en comparación con la atresia auricular congénita $(21,22)$. En nuestro estudio, se observó que solo 2 casos presentaron estenosis y colesteatoma concomitante: en uno de los casos la estenosis estaba asociada una microtia grado 4, mientras que el otro correspondía a un paciente de 70 años de edad, en el que el largo tiempo de evolución podría haber contribuido al desarrollo del colesteatoma. Estudios poblacionales, realizados en Europa y Estados Unidos de América, muestran una prevalencia entre 0,83 y 4,34 por 10,000 nacimientos (23). En México, el registro y vigilancia epidemiológica de malformaciones congénitas externas ha reportado una prevalencia de 7,37/10,000 nacidos vivos y muertos durante el periodo 1978-2010, más alta que la reportada en otras poblaciones (24).

En América Latina se tiene asimismo una alta prevalencia de microtia: en Chile, 5, 2 por 10,000 (10); y en Argentina, 4,41 por cada 10,000 nacimientos, tantos vivos como muertos (25). Si bien en el presente estudio se encontró un prevalencia baja (0,14 por 10,000 nacidos vivos), se considera que esta cifra podría aumentar significativamente teniendo en cuenta que las historias clínicas utilizadas fueron solamente las quirúrgicas, no contando con aquellos casos que acudieron a consultorio externo. Por lo tanto, aquí podría hallarse un sesgo por falta de información.

Por su parte, las disgenesias de la cadena osicular en sus diferentes grados, suelen ser muy poco frecuentes. Frente a una hipoacusia uni o bilateral, no progresiva, que se presenta en la infancia o la adolescencia con otoscopia normal, exámenes audiológicos compatibles con hipoacusia conductiva o mixta a predomino conductivo e impedanciometría con curva de rigidez 
o interrupción de cadena, se debe sospechar de una malformación congénita de la cadena osicular, lo cual se plantea el diagnóstico diferencial con otras patologías otológicas, especialmente con otoesclerosis (26). También es importante determinar si es necesario el uso de auxiliares auditivos para no mermar el desarrollo del lenguaje en el paciente. En la presente investigación se encontró un solo caso de disgenesia de la cadena osicular en un paciente de sexo masculino de 20 años.

Finalizando, tomando en consideración los resultados de la presente investigación, se concluye que resulta importante tanto para el pediatra como para el otorrinolaringólogo lograr identificar precozmente las malformaciones congénitas del oído, para así poder instaurar terapéuticas oportunas y acertadas, que mejoren ampliamente la calidad de vida de los pacientes. Esta capacidad de identificación vendrá dada por un adecuado conocimiento de la embriología, la cual constituye la piedra angular para poder comprender el origen y la relación que existe entre las malformaciones del oído externo y medio y es una ciencia en la que debe insistirse ya a partir de la formación de grado.

Nunca está de más recalcar que el paciente debe ser examinado en su totalidad y que no se debe pasar por alto la presencia de otras malformaciones o patologías concomitantes tanto a nivel sistémico como dentro del área otorrinolaringológica, teniendo en cuenta que muchas veces es mejor pecar por exceso que por defecto.

\section{CONFLICTOS DE INTERÉS Y FUENTE DE FINANCIACIÓN}

Los autores declaran no poseer conflictos de interés. Fuente de financiación: ninguna.

\section{REFERENCIAS BIBLIOGRÁFICAS}

1. Bellucci RJ.Congenital aural malformations: diagnosis and treatment. Otolaryngol Clin North Am. 1981;14(1):95-124. URL.

2. Adams A, Mankad K, Offiah C, Childs L. Branchial cleft anomalies: a pictorial review of embryological development and spectrum of imaging findings. Insights Imaging. 2016;7(1):69-76. http://dx.doi.org/10.1007/s13244-015-0454-5

3. Carlson B, Kantaputra P. Embriología humana y biología del desarrollo. 5a ed. Barcelona: Elsevier; 2014.

4. Teissier N, Bernard S, Quesnel S, Van Den Abbeele T. Audiovestibular consequences of congenital cytomegalovirus infection. European Annals of Otorhinolaryngology, Head and Neck Diseases 2016; 133(6):413-418. http://dx.doi.org/10.1016/j.anorl.2016.03.004

5. Bartel-Friedrich S, Wulke C. Classification and diagnosis of ear malformations. GMS Curr Top Otorhinolaryngol Head Neck Surg. 2007; 6: Doc05. URL.

6. Zhao H, Ma L, Qi X, Qin J, Yin B, Zhong M, et al. A Morphometric Study of the Newborn Ear and an Analysis of Factors Related to Congenital Auricular Deformities. Plast Reconstr Surg. 2017;140(1):147-155. http://dx.doi.org/10.1097/PRS.0000000000003443

7. Ji S, Ou Y, Xu Y, Zheng Y. The influence of congenital malformation of external and middle ear on the psychologic status of children patients. Lin Chung Er Bi Yan Hou Tou Jing Wai Ke Za Zhi. 2014;28(7):450-452. URL. 
8. Grundfast KM, Camilon F: External auditory canal stenosis and partial atresia without associated anomalies. Ann Otol Rhinol Laryngol. 1986: 95(5 Pt 1):505-509. http://dx.doi.org/10.1177/000348948609500512

9. BW Jafek, GT Nager, J Strife, Gayler RW. Congenital aural atresia: an analysis of 311 cases. Trans Sect Otolaryngol Am Acad Ophthalmol Otolaryngol. 1975;80(6):588-595. $\underline{U R L}$.

10. Nazer J, Lay-Son G, Cifuentes L. Prevalencia de nacimiento de microtia-anotia. Maternidad del Hospital Clínico de la Universidad de Chile, período 1983-2005. Rev Méd Chile 2006;134:1295-1301. http://dx.doi.org/10.4067/S0034-98872006001000012

11. Daniali LN, Rezzadeh K, Shell C, Trovato M, Ha R, Byrd HS. Classification of Newborn Ear Malformations and their Treatment with the EarWell Infant Ear Correction System. Plast Reconstr Surg. 2017;139(3):681-691. http://dx.doi.org/10.1097/PRS.0000000000003150

12. Rimbau Serrano I, Molina Gutiérrez MA. Tumoración en la región anterior del hélix: fístula preauricular. Rev Pediatr Aten Primaria. 2016;18:179-181. URL.

13. Lee K, Woo S, Kim S, Yang J, Cho Y. The Prevalence of Preauricular Sinus and Associated Factors in a Nationwide Population-Based Survey of South Korea. Otol Neurotol. 2014;35(10):1835-1838. http://dx.doi.org/10.1097/MAO.0000000000000587

14. Baatenburg de Jong R. A new surgical technique for treatment of preauricular sinus. Surgery. 2005;137(5):567-570. URL.

15. Melnick M, Myrianthopoulos NC N, Paul. N. External ear malformations: epidemiology, genetics, and natural history. Birth Defects Orig Artic Ser. 1979;15(9):i-ix, 1-140. URL.

16. Quintana Díaz JC, Miranda Tarragó JD, Quintana Giralt M. Comportamiento del quiste branquial en pacientes del Hospital General Docente "Ciro Redondo García", Artemisa, 1993-2009. Revista Cubana de Estomatología 2012;49(1):39-46. URL.

17. Medina Vega LD, Márquez Rancaño E, Concepción Guzmán J, Rodríguez Jiménez R, Martín Pino J. Correlación clínico-citohistológica de los quistes congénitos cervicales. Rev Esp Cirug Oral y Maxilofac 2004;26:22-28. URL.

18. Estrada Sarmientos M. Quistes y fístulas del conducto tirogloso. Nuestra experiencia. Rev Cubana Cir 1996;35: 138-44. URL.

19. Santana Garay JC. Alteraciones del desarrollo de la cara, la boca y el cuello. En: Santana Garay JC. Atlas de Patología del Complejo bucal. 1a ed. Ciudad de la Habana: CientíficoTécnica; 1985. p. 20-24.

20. Raspall G, Arcas A. Adenopatías Cervicales en oncología. Clínica, diagnóstico y tratamiento. Medicina Oral 2000; 5:89-95. URL.

21. Mayer TE, Brueckmann H, Siegert R, Witt A, Weerda H. High-resolution CT of the temporal bone in dysplasia of the auricle and external auditory canal. AJNR Am J Neuroradiol. 1997;18(1):53-65. URL.

22. Kosling S, Omenzetter M, Bartel-Friedrich S. Congenital malformations of the external and middle ear. Eur J Radiol 2009;69(2):269-279. URL.

23. Canfield M, Langlois $P$, Nguyen L, Scheuerle A. Epidemiologic features and clinical subgroups of anotia/microtia in Texas. Birth Defects Res A Clin Mol Teratol. 2009;85(11):905-13. http://dx.doi.org/10.1002/bdra.20626

24. Aguinaga-Ríos M, Frías S, Arenas-Aranda DJ, Morán-Barroso VF. Microtia-atresia: aspectos clínicos, genéticos y genómicos. Bol Med Hosp Infant Mex 2014;71:387-395. http://dx.doi.org/10.1016/j.bmhimx.2014.11.001 
25. Campaña H, Pawluka MS, López Camel JS, Grupo de Estudio del ECLAMC. Prevalencia al nacimiento de 27 anomalías congénitas seleccionadas, en 7 regiones geográficas de la Argentina. Arch Argent Pediatr 2010;108(5):409-417. URL.

26. Zambrana H, De Maio S, Garcia Delgado P. La disgenesia de la cadena osicular. A propósito de un caso (hallazgo cadavérico); Revista FASO 2015;22(3):28-31. URL. 